\title{
Magnetic Patterning by Electron Beam-Assisted Carbon Lithography
}

\author{
Pietro Genoni, ${ }^{\dagger}$ Francesca Genuzio, ${ }^{\ddagger}$ Tevfik Onur Menteş, ${ }^{\ddagger}$ Benito Santos, ${ }^{\ddagger}$ Alessandro Sala, ${ }^{\ddagger}, \perp_{\mathbb{C}}$ \\ Cristina Lenardi, ${ }^{\dagger}$ and Andrea Locatelli* ${ }^{*} \neq$ (1) \\ ${ }^{\dagger}$ CIMAINA, Department of Physics, Università degli Studi di Milano, via Celoria 16, I-20133 Milan, Italy \\ ${ }^{\ddagger}$ Elettra-Sincrotrone Trieste, S.S. $14 \mathrm{~km} 163.5$ in AREA Science Park, Basovizza, I-34149 Trieste, Italy \\ ${ }^{\S}$ Department of Physics, Università degli Studi di Trieste, via Valerio 2, I-34127 Trieste, Italy
}

\section{Supporting Information}

ABSTRACT: We report on the proof of principle of a scalable method for writing the magnetic state by electronstimulated molecular dissociative adsorption on ultrathin Co on $\operatorname{Re}(0001)$. Intense microfocused low-energy electron beams are used to promote the formation of surface carbides and graphitic carbon through the fragmentation of carbon monoxide. Upon annealing at the $\mathrm{CO}$ desorption temperature, carbon persists in the irradiated areas, whereas the clean surface is recovered elsewhere, giving origin to chemical patterns with nanometer-sharp edges. The accumulation of carbon is found to induce an in-plane to out-of-plane spin reorientation transition in Co, manifested by the appearance of striped magnetic domains. Irradiation at doses in excess of 1000 $\mathrm{L}$ of $\mathrm{CO}$ followed by ultrahigh vacuum annealing at $380{ }^{\circ} \mathrm{C}$ determines the formation of a graphitic overlayer in the irradiated areas, under which Co exhibits out-of-plane magnetic anisotropy. Domains with opposite magnetization are separated here by chiral Neél walls. Our fabrication protocol adds lateral control to spin reorientation transitions, permitting to tune the magnetic anisotropy within arbitrary regions of mesoscopic size. We envisage applications in the nano-engineering of graphene-spaced stacks exhibiting the desired magnetic state and properties.

KEYWORDS: spin reorientation transition, cobalt thin films, magnetic lithography, patterning, chiral domain walls

\section{INTRODUCTION}

The ability to create magnetic nanopatterns, that is, controlled lateral modulations in the magnetic anisotropy (MA) of a continuous magnetic film, is key to the development of devices and sensors with novel architecture and functionality. ${ }^{1}$ Magnetic patterning is often carried out by means of ion irradiation combined with conventional lithography and etching and permits lateral resolution nowadays nearing the nanometer. ${ }^{2}$ The effective MA of the magnetic medium is controlled through the energy and fluence of ions, which can induce structural transitions ${ }^{3,4}$ and disorder at the film interfaces $^{5-7}$ or can change the chemical state of the material. ${ }^{8}$ Irradiation and implantation methods, however, have a limited field of application because they can produce irreversible structural damage. Varied techniques have been proposed as an alternative, for example, thermally assisted scanning probe lithography ${ }^{9}$ or electron irradiation ${ }^{10,11}$ to name just a few.

The usage of e-beams for magnetic patterning was pioneered by Allenspach and co-workers. They studied ultrathin Co films on $\operatorname{Pt}(111)$ and found that irradiation with high-energy electrons induces an in-plane to out-of-plane spin reorientation transition (SRT), which was attributed to a structural transformation of the magnetic medium. ${ }^{10}$ Surprisingly, ebeam-induced magnetic phenomena have remained substantially unexplored since then, with only a few exceptions noted.
For instance, electron irradiation was found to induce the switching of a metastable state in $\mathrm{Fe}$ thin films grown on GaAs(110), producing an in-plane to in-plane SRT. This phenomenon was explained by invoking electronic excitations and ruling out adsorbate effects. ${ }^{11}$ On the other hand, a recent study attributed an in-plane to in-plane SRT occurring in ultrathin $\mathrm{Co}(110)$ to the electron-stimulated irradiation and desorption of the adsorbed carbon monoxide, ${ }^{12}$ which permitted to demonstrate magnetic patterning at the millimeter scale. ${ }^{13}$ Yet, a microscopic proof of this concept is still missing and its mechanisms certainly deserve further investigation.

As a novel aspect pertinent to this subject, we address here the effect that stimulated molecular dissociative adsorption induces on thin-film magnetism, focusing on the model case of $\mathrm{CO}$ on cobalt. Indeed, electron-beam-stimulated processes involving simple molecular adsorbates have attracted huge attention in the past decades, both experimentally and theoretically. ${ }^{14,15}$ Yet, these studies mainly focused on the fundamental physical mechanisms of molecular fragmentation, leaving basically unexplored the territory of magnetism. In the

Received: May 14, 2018

Accepted: July 18, 2018

Published: July 18, 2018 
following, we will describe a method to graft micrometer-sized carbon patterns inducing an in-plane to out-of-plane SRT in few-layer hcp Co on $\operatorname{Re}(0001)$. By irradiating the film surface in $\mathrm{CO}$ ambient using microfocused beams, we will show that monolayer (ML) atomic carbon can be accumulated and, most remarkably, converted to a single graphitic layer upon a mild annealing treatment. As we will see, these carbon overlayers can greatly influence the MA of the underlying Co thin film.

Our work is motivated by the current strong interest in magnetic heterostacks comprising a two-dimensional (2D) nonmagnetic material as a spacer layer because of their relevance for spintronics. ${ }^{1}$ As a prototypical $2 \mathrm{D}$ material, graphene is ideally suited for this type of application as it enhances perpendicular MA (PMA) in Co thin films, ${ }^{16}$ indeed a very desirable characteristic for most magnetic memory devices. Notably, graphene-spaced magnetic stacks can be considered as the essential building blocks for the synthesis of ferromagnetic (FM) and antiferromagnetic (AFM) materials with tunable magnetic properties. In this regard, antiferromagnetic exchange coupling was recently demonstrated in $\mathrm{Fe} /$ graphene/Co layers supported on $\operatorname{Ir}(111) .{ }^{17}$ As a further reason of interest, we note that graphene/Co is currently under the spotlight owing to the observation of chiral spin textures at the graphene-ferromagnetic metal interface, their origin being attributed to the Rashba effect. ${ }^{18}$ The capability to locally deposit $2 \mathrm{D}$ carbon layers is thus highly desirable. As will be shown, our method does not require high temperatures, which, together with lithographic capability, is a notable advantage over chemical vapor deposition (CVD) techniques.

\section{EXPERIMENTAL SECTION}

All experiments were carried out at the "Nanospectroscopy" beamline of the Elettra Synchrotron (Trieste, Italy), using the spectroscopic photoemission and low-energy electron microscope (SPELEEM). ${ }^{19}$ This microscope allows us to correlate the magnetic state of the Co film, probed by X-ray magnetic circular dichroism photoemission electron microscopy (XMCD-PEEM), ${ }^{20,21}$ to its surface stoichiometry and electronic structure, characterized by means of laterally resolved $\mathrm{X}$-ray photoelectron spectroscopy (XPS) and microprobe angleresolved photoelectron spectroscopy ( $\mu$-ARPES). Crucial to our work, the SPELEEM can also be operated as a LEEM, permitting us not only to monitor the growth and structural quality of the cobalt films, but also to perform micro-spot irradiation with its electron source. In the LEEM, the specimen is probed with a microfocused, collimated electron beam emitted by an $\mathrm{LaB}_{6}$ cathode. The microscope then collects the electrons that are elastically backscattered at the specimen surface, which can be imaged in either real or reciprocal space (see the Methods Section).

The model system chosen for this work is affine to the thoroughly studied $\mathrm{Co} / \mathrm{Ru}(0001)$, sharing with it the same substrate symmetry (hcp structure at room temperature; see the Methods Section) and similar lattice constant. It is important to note that ultrathin Co films frequently display SRTs and other intriguing magnetic configurations. ${ }^{20}$ In-plane to out-of-plane and out-of-plane to in-plane SRTs were observed for hcp $\mathrm{Co} / \mathrm{Ru}(0001)$, occurring at thicknesses of 2 and 3 atomic layers (ALs), respectively. ${ }^{22}$ Further, we note that the MA of ultrathin Co films is known to be strongly influenced by the adsorption of $\mathrm{CO}$ and other gases, as has been repeatedly reported. ${ }^{23-26}$ The choice of a heavy metal substrate, Re, was functional to obtaining strong and asymmetric exchange interactions at the interface, also known as the Dzyaloshinskii-Moriya interaction (DMI). ${ }^{27,28}$

Ultrathin Co films of homogeneous thickness in the range 4-6 AL were grown as detailed in the Methods Section and section S1 of the Supporting Information. At the thicknesses hereby considered, that is, greater than or equal to $4 \mathrm{AL}, \mathrm{X}$-ray circular dichroic asymmetries up to $15 \%$ were observed at the Co $\mathrm{L}_{3}$ edge. This large dichroic effect indicates predominantly in-plane MA and is rationalized considering that the synchrotron light impinges on the sample at an angle of incidence of $16^{\circ}$ from the surface plane. Our observations are in agreement with previous measurements on the closely related Co/ $\mathrm{Ru}(0001)$ system, which was found to exhibit in-plane MA at thicknesses equal to or greater than $3 \mathrm{AL}^{22}$ Yet, it has to be noted that XMCD-PEEM cannot easily quantify canted configurations, which were previously observed for Co films grown on W(110) at a thickness in the range 3-8 MLs. ${ }^{29}$ Nonetheless, on the basis of image contrast considerations, we can safely exclude large tilt angles in the as-grown Co film.

\section{CONCEPT OF THE PATTERNING METHOD}

The protocol underlying chemomagnetic patterning is illustrated in Figure 1. In the first step (top panel), the clean

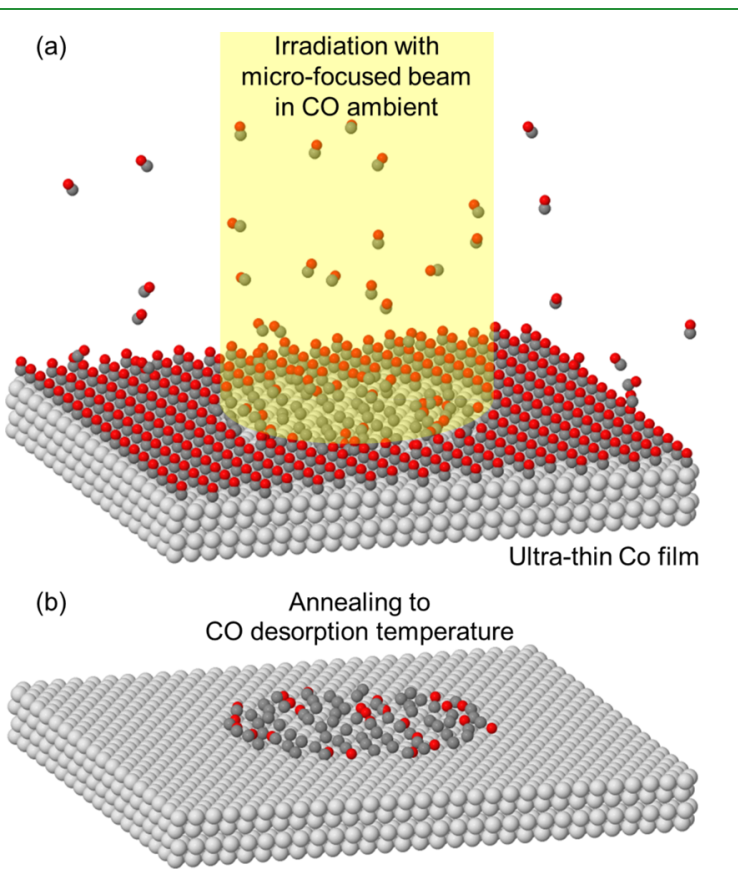

Figure 1. Scheme of the grafting protocol used to produce chemomagnetic patterns in ultrathin Co. (a) The film is exposed to $\mathrm{CO}$ at room temperature and irradiated with a focused electron beam, so that the dissociative adsorption of the molecule is induced. (b) The sample is annealed at $170{ }^{\circ} \mathrm{C}$ to desorb molecularly adsorbed $\mathrm{CO}$ from the nonirradiated surface regions.

Co film (showing in-plane MA) is saturated with $\mathrm{CO}$ and then irradiated with an intense, microfocused electron beam while being exposed to $\mathrm{CO}$ at room temperature. The irradiation with low-energy electrons induces $\mathrm{CO}$ dissociative adsorption and desorption, as has been amply documented for varied transition metals. ${ }^{15}$ As shown previously, the energy threshold for electron and photon $\mathrm{CO}$ dissociation is close to $15 \mathrm{eV}$; the largest cross section of the electron-stimulated process is reached in the energy range $50-100 \mathrm{eV}^{30,31}$ The e-beam irradiation is carried out in $\mathrm{CO}$ ambient at pressures in the range $10^{-9}$ to $10^{-6} \mathrm{mbar}$, so that molecular adsorption can counterbalance the effect of stimulated desorption. This permits the accumulation of $\mathrm{C}$ on the surface, as previously reported by Lambert and Comrie. ${ }^{32}$ We also note that, at the $\mathrm{CO}$ pressure considered here, molecular fragmentation mostly occurs on the surface owing to the long resident time of $\mathrm{CO}$ following adsorption. Negligible dissociation occurs in the gas 
phase, the $\mathrm{CO}$ density being far too low to allow ionizing a significant number of molecules.

In the second step (Figure 1, bottom panel), ultrahigh vacuum (UHV) conditions are restored; the sample is then annealed above the $\mathrm{CO}$ desorption temperature, that is, 170 ${ }^{\circ} \mathrm{C} .{ }^{33}$ In this manner, molecularly adsorbed $\mathrm{CO}$ is removed from the nonirradiated regions. These regionstherefore exhibit in-plane MA, just like the as-grown film. On the other hand, atomic carbon cannot be removed from the regions previously irradiated with the e-beam. In this respect, it is important to note that the bond strength of carbidic $\mathrm{C}$ to $\mathrm{Co}(0001)$ is much larger $(6.47 \mathrm{eV} /$ atom $)$ than that of chemisorbed $\mathrm{CO}(1.46 \mathrm{eV} /$ atom). ${ }^{34}$ As will be shown below, both carbidic and graphitic carbon layers have a strong influence on the magnetic properties of the Co film, thereby inducing in-plane to outof-plane SRTs. Clearly, the concept hereby illustrated can be generalized, for instance, using other molecules in place of $\mathrm{CO}$, provided they do not thermally dissociate during the annealing treatment.

\section{RESULTS AND DISCUSSION}

4.1. Irradiation at Low CO Doses. Figure 2 shows roomtemperature LEEM and XMCD-PEEM images of circular structures grafted on $4 \mathrm{AL}$ thick Co films grown on $\operatorname{Re}(0001)$. All structures shown in Figure 2a were fabricated by predosing $2.5 \mathrm{~L}$ of $\mathrm{CO}$ before irradiation and then delivering further $4.5 \mathrm{~L}$ during irradiation, as previously detailed. The four smallest disks measure about $500 \mathrm{~nm}$ in diameter. LEEM imaging conditions were adjusted so that the irradiated spots appear brighter than their surroundings, exploiting differences in the low-energy electron reflectivity that are related to the modified chemical composition of the topmost surface layer. The XMCD-PEEM image in Figure $2 b$ illustrates the magnetic state of the region corresponding to the disks highlighted by the dashed contour in Figure 2a. Striped magnetic domains are clearly visible within the irradiated areas, indicating the onset of an in-plane to out-of-plane SRT. As seen in other experiments, the $\mathrm{CO}$ dose of $4.5 \mathrm{~L}$ is barely sufficient to induce the transition, so that the XMCD image depicts the magnetic state very close to the transition critical point.

The LEEM and XMCD-PEEM images in Figure 2c,d display a micrometer-sized disk that was printed by irradiating the cobalt surface for a longer time (a dose of about $10 \mathrm{~L}$ of $\mathrm{CO}$ was given during irradiation). The LEEM image demonstrates that the disk exhibits quite sharp edges. A cross-sectional cut of the LEEM image across the disk edge is shown in Figure 2e, along with a step function fit that reveals an edge width of less than $30 \mathrm{~nm}$. This broadening results from both the limited resolution of the microscope and the sample drift during irradiation. As can be seen in the XMCD-PEEM image in Figure $2 \mathrm{~d}$, the stripe pattern is fully established at this stage. An intensity profile perpendicular to the stripe direction reveals a period of about $120 \mathrm{~nm}$ (see Figure 2f).

The emergence of a periodic stripe pattern is a clear, unambiguous sign of an in-plane to out-of-plane SRT. ${ }^{35,36}$ The out-of-plane MA observed within the irradiated areas is confirmed by the decrease of the magnetic contrast due to the measurement geometry. ${ }^{37}$ Overall, we observed the SRT in Co thicknesses of up to $6 \mathrm{AL}$. Our XMCD data show clearly that the stripe period increases with increasing $\mathrm{CO}$ dose. As will be shown below, this process eventually leads to the formation of very large, stationary domains, as is expected when moving away from the critical point of the transition. ${ }^{35}$
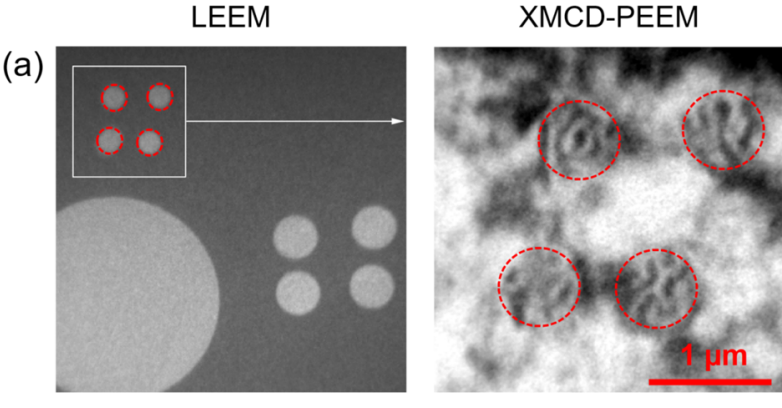

(b) (c)

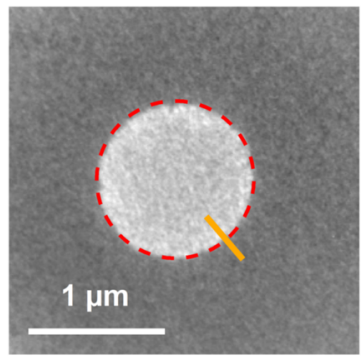

(e)

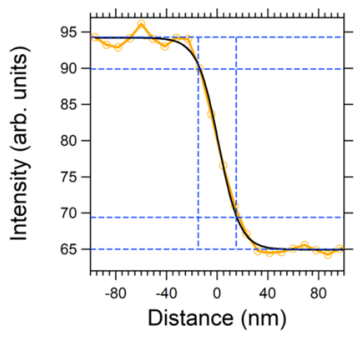

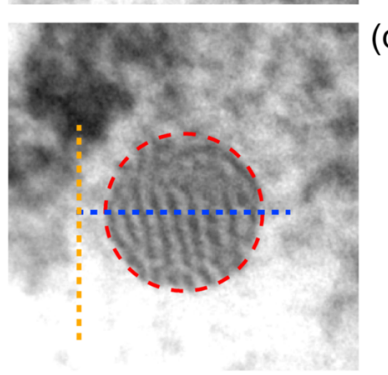

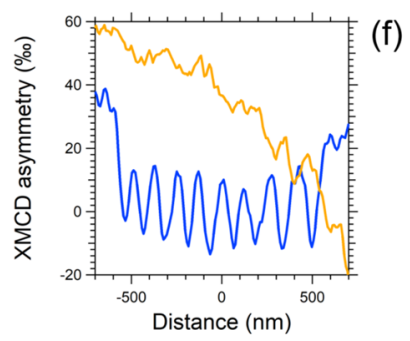

Figure 2. Images of chemomagnetic patterns on a Co film with a homogeneous thickness of $4 \mathrm{AL}$; all structures were grafted upon irradiation with $50 \mathrm{eV}$ electrons at $p_{\mathrm{CO}}$ of $2 \times 10^{-8} \mathrm{mbar}$. The e-beam flux density on the sample was $1.3 \times 10^{16} \mathrm{e}^{-} \mathrm{cm}^{-2} \mathrm{~s}^{-1}$. (a) LEEM image at $V_{\mathrm{S}}=8 \mathrm{~V}$ of nine e-beam irradiated disks of diameter approximately 5, 1, and $0.5 \mu \mathrm{m}$. CO dose: $4.5 \mathrm{~L}$; (b) Co $\mathrm{L}_{3}$ edge XMCD-PEEM image of the region highlighted by the white box in (a). (c) LEEM image at $V_{\mathrm{S}}=9 \mathrm{~V}$ of an e-beam irradiated disk measuring approximately $1 \mu \mathrm{m}$ in diameter; CO dose: $9.75 \mathrm{~L}$; (d) XMCD-PEEM image of the same region at the Co $\mathrm{L}_{3}$ edge. (e) Intensity profile across the orange line in the LEEM image in (c) and fit using a step function convoluted with a Gaussian of full width at half-maximum of $30 \mathrm{~nm}$. The dashed blue lines indicate the 15-85\% distance between minimum and maximum intensity. (f) Intensity profiles across the blue and orange dashed lines in the XMCD-PEEM image in (d). The plot highlights a difference in dichroic contrast of $\sim 3.5$ between the irradiated disk and its surroundings. The magnetic stripes exhibit regular oscillations with a period of about $120 \mathrm{~nm}$.

Temperature-dependent XMCD-PEEM measurements showed that the stripe patterns are visible up to about 140 ${ }^{\circ} \mathrm{C}$, a large enough value to ensure sufficient stability to the magnetic state observed at room temperature. The large XMCD contrast recorded outside the irradiated disks indicates that the magnetization is still oriented in-plane in the nonirradiated regions.

The chemical state of the adlayer was investigated by probing the $\mathrm{C} 1 \mathrm{~s}$ and $\mathrm{O} 1 \mathrm{~s}$ core-level emission inside and outside disks with a diameter of $5 \mu \mathrm{m}$. The measurements were carried out at room temperature, after irradiation in $\mathrm{CO}$ ambient $\left(P_{\mathrm{CO}}=2.5 \times 10^{-8} \mathrm{mbar}\right)$ and UHV annealing. Selected XPEEM images of an irradiated spot are shown in Figure 3a,b. They qualitatively demonstrate the different surface stoichiometry inside and outside the irradiated disk. These differences are quantitatively assessed by the spectra 


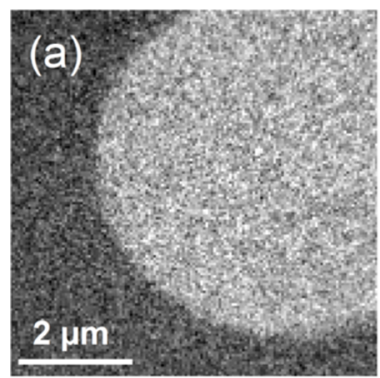

XPEEM C $1 s$

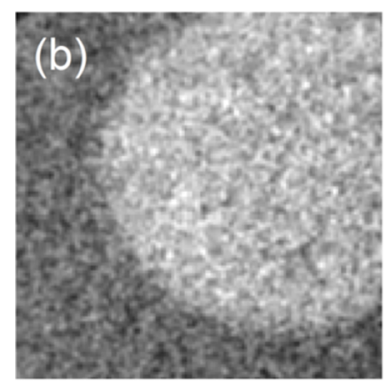

XPEEM O $1 s$
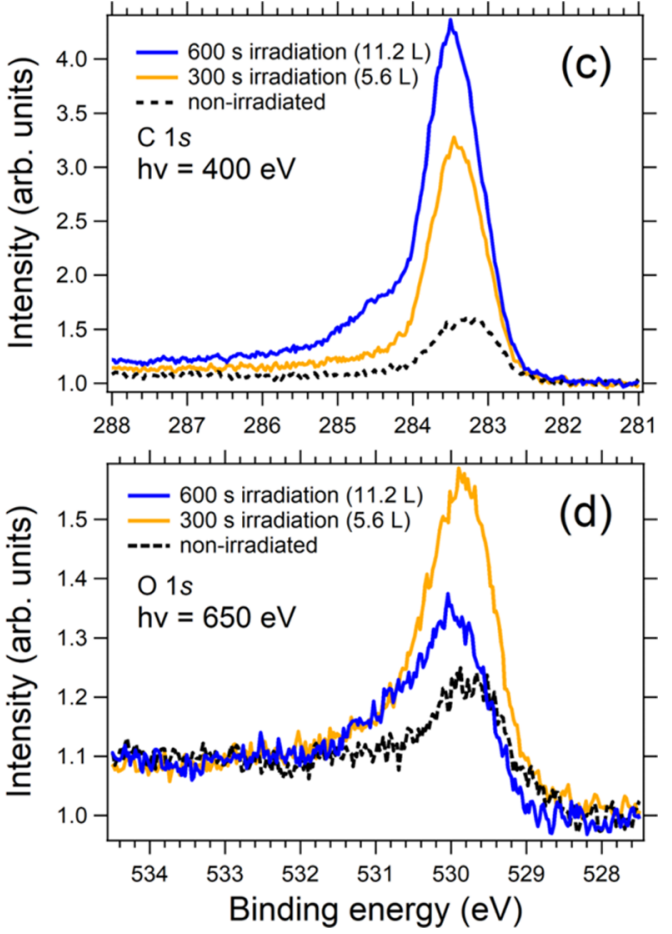

Figure 3. (a,b) C $1 \mathrm{~s}(h \nu=400 \mathrm{eV})$ and $\mathrm{O} 1 \mathrm{~s}(h \nu=650 \mathrm{eV})$ XPEEM images of a $5 \mu \mathrm{m}$ diameter disk, printed by e-beam irradiation in CO ambient. $\mathrm{CO}$ dose: $6.75 \mathrm{~L}$. The images demonstrate carbon and oxygen accumulation within the e-beam irradiated region. $(\mathrm{c}, \mathrm{d}) \mathrm{C} 1 \mathrm{~s}(h \nu=400 \mathrm{eV})$ and $\mathrm{O}$ $1 \mathrm{~s}(h \nu=650 \mathrm{eV})$ spectra from the irradiated disks and the nonirradiated surface region surrounding it. The $\mathrm{C}$ enrichment reflects the $\mathrm{CO}$ dose. For explanation, see text.

Table 1. Summary of the results reported in this work $^{a}$

\begin{tabular}{|c|c|c|c|c|c|c|c|c|}
\hline \multirow[b]{2}{*}{$d_{\mathrm{Co}}(\mathrm{AL})$} & \multirow[b]{2}{*}{ dose $(L)$} & \multirow[b]{2}{*}{ UHV annealing treatment } & \multicolumn{3}{|c|}{ irradiated area } & \multicolumn{3}{|c|}{ non-irradiated surface } \\
\hline & & & $\theta_{\mathrm{C}}\left(\mathrm{eML}_{\mathrm{gr}}\right)$ & C type & MA & $\theta_{\mathrm{C}}\left(\mathrm{eML}_{\mathrm{gr}}\right)$ & C type & MA \\
\hline 4.0 & 4.5 & $5^{\prime} 190{ }^{\circ} \mathrm{C}$ & 0.12 & carbidic & $\uparrow$ & 0.04 & carbidic & $\rightarrow$ \\
\hline $4-5$ & 5.6 & $5^{\prime} 190^{\circ} \mathrm{C}$ & 0.14 & carbidic & $\uparrow$ & 0.04 & carbidic & $\rightarrow$ \\
\hline $4-6$ & 11.2 & $5^{\prime} 190^{\circ} \mathrm{C}$ & 0.23 & carbidic & $\uparrow$ & 0.04 & carbidic & $\rightarrow$ \\
\hline $4-5$ & $>10^{3}$ & ramp to $380{ }^{\circ} \mathrm{C}$ & 0.77 & graphitic & $\uparrow$ & 0.08 & carbidic & $\rightarrow$ \\
\hline 4.7 & $>10^{3}$ & $5-10^{\prime} 400{ }^{\circ} \mathrm{C}$ & 0.77 & graphitic & $\uparrow$ & Co islanding & & $\rightarrow$ \\
\hline
\end{tabular}

${ }^{a}$ The $\mathrm{C}$ coverage $\theta_{\mathrm{C}}$, overlayer chemical state (type of carbon), and MA of the irradiated and nonirradiated surface regions are listed as a function of cobalt thickness $\left(d_{\mathrm{Co}}\right)$, CO dose delivered during e-beam irradiation $(\mathrm{L})$, and the final temperature and duration of the annealing treatment. Legend: $\rightarrow$ indicates in-plane MA, $\uparrow$ indicates out-of-plane MA.

shown aside. As can be seen in Figure $3 c$, the intensity of the $\mathrm{C}$ 1s emission increases with the $\mathrm{CO}$ dose delivered during ebeam irradiation. In all spectra, the component at binding energy (BE) of $283.5 \mathrm{eV}$ is clearly dominant and can be attributed to carbidic species. ${ }^{38}$ A careful inspection of the spectrum obtained after $10 \mathrm{~min}$ irradiation reveals a second component, centered at $284.9 \mathrm{eV}$, readily ascribed to the formation of $\mathrm{sp}^{2}$ carbon and thus graphitic in nature. ${ }^{39}$

The adlayer coverage was estimated by evaluating the area of the measured $\mathrm{C}$ 1s spectrum and comparing it with that of single-layer graphene grown by high-temperature CVD on a thick Co film supported on W(110). We determined a carbon coverage of 0.14 and $0.23 \mathrm{eML}_{\mathrm{gr}}$ (graphene-equivalent $\mathrm{ML}$ ) for the disks irradiated for 5 and 10 minutes, respectively. On the other hand, the nonirradiated part of the surface, still showing in-plane MA, exhibits a significantly lower $\mathrm{C}$ coverage, about $0.04 \mathrm{eML}_{\mathrm{gr}}$, which is compatible with the surface being contaminated by the background pressure of $\mathrm{CO}$ and other hydrocarbons during the time needed to prepare and perform the measurements. We thus estimate that the amount of $\mathrm{C}$ necessary to induce the SRT is very close to $0.1 \mathrm{eML}_{\mathrm{gr}}$. Results for the different preparations reported in this study are summarized in Table 1. These findings demonstrate a clear tendency toward the accumulation of carbon under X-ray irradiation in $\mathrm{CO}$ ambient. As a matter of fact, graphitization processes resulting from $\mathrm{CO}$ dissociation occur on all surfaces that are exposed to low-energy electrons beams, extreme UV radiation, and soft X-rays. ${ }^{40}$ As is well known in the X-ray optics community, they lead to detrimental effects, affecting mirror reflectivity at and above the carbon $\mathrm{K}$ absorption edge. $^{41,42}$

Focusing now on $\mathrm{O}$ 1s spectra in Figure 3d, the main peak $(529.6 \mathrm{eV})$ is assigned to the adsorbed atomic oxygen bound to cobalt, forming $\mathrm{CoO}$ species. ${ }^{43}$ We ascribe the tail at higher $\mathrm{BE}$ to the formation of a disordered oxygen adlayer, with multiple adsorption sites becoming populated. ${ }^{44}$ The absence of emission at $532 \mathrm{eV}$, however, indicates that no molecular $\mathrm{CO}$ is adsorbed on the surface; that is, $\mathrm{CO}$ has already undergone dissociative adsorption or desorption. Interestingly, the $\mathrm{O} 1 \mathrm{~s}$ emission reaches an intensity maximum correspond- 

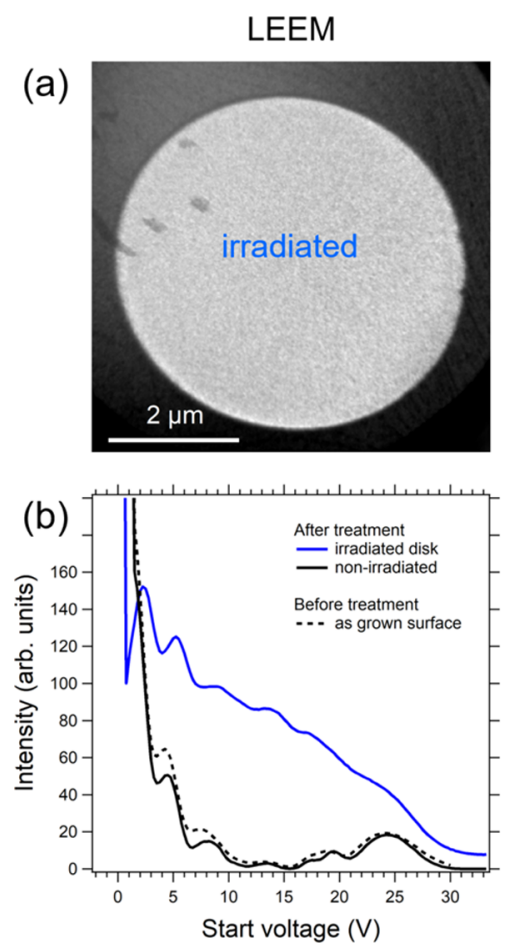

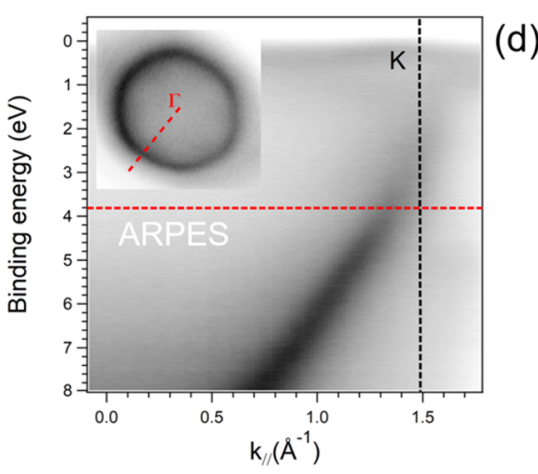

Figure 4. (a) LEEM image of an e-beam irradiated $5 \mathrm{AL}$ thick Co film after annealing to $380{ }^{\circ} \mathrm{C}$. A total CO dose of $2700 \mathrm{~L}$ was delivered during irradiation with $60 \mathrm{eV}$ electrons. The small dark patches (top left) are surface regions that were not fully covered during the deposition of the first Co layer. Their thickness is $4 \mathrm{AL}$ of Co. (b) LEEM-IV spectra inside and outside (blue and black curves, respectively) the irradiated disk. Note that the low-energy electron reflectivity of the surface does not change upon treatment ( $\mathrm{CO}$ exposure followed by annealing for the nonirradiated regions). (c) XPEEM C 1s spectra from inside (blue line) and outside (black dashed line) another e-beam irradiated structure (a CO dose of 1350 $\mathrm{L}$ was delivered in this case); the C 1s spectrum (orange) of CVD graphene grown on the same substrate is shown for comparison. The XPEEM image in the inset represents the C map; photon energy: $400 \mathrm{eV}$. (d) $\mu$-ARPES data from inside the irradiated disk shown in (c). The graph is a cross-sectional cut along the radial direction indicated by the dashed red line in the pattern shown in the inset. The position of the $K$ point is indicated by the black dashed line, whereas the horizontal red dashed line indicates the energy at which the pattern shown in the inset was acquired. The $\pi$ band of rotationally incoherent graphene can be clearly recognized. Photon energy: $40 \mathrm{eV}$.

ing to the dose of $5 \mathrm{~L}$ of $\mathrm{CO}$ and notably decreases for higher $\mathrm{CO}$ doses. This observation is consistent with complementary photon-stimulated desorption experiments that we performed, indicating that the desorption channel becomes progressively more important as $\mathrm{C}$ accumulates on the surface. The decrease of the $\mathrm{O} 1 \mathrm{~s}$ signal is attributed to an attenuation effect due to the growing carbon layer. Finally, we estimate the ratio between the oxygen and carbon coverages by computing the areas of $\mathrm{C} 1 \mathrm{~s}$ and $\mathrm{O} 1 \mathrm{~s}$ peaks (in this case, both spectra were measured at the same photon energy of $650 \mathrm{eV}$ ), taking into account the photoionization cross sections of the corresponding core levels and the energy-dependent transmission of the spectrometer. We obtain a value of $\sim 1 / 3$, which corresponds to an oxygen coverage of less than $0.1 \mathrm{eML}_{\mathrm{Co}}$ ( $\mathrm{ML}$ equivalent of $\mathrm{Co}$ ).

We briefly mention that the magnetic patterns can be preserved from the action of external agents, for example, oxidation, a crucial aspect for the fruition of our patterning method. This was achieved through the deposition of a thin $\mathrm{Ag}$ film (with thickness of $\sim 6 \mathrm{AL}$ ) above $\mathrm{Co}$. Ag was chosen because it maintains in-plane $\mathrm{MA}$ in the nonirradiated regions. ${ }^{45}$ Layer-by-layer growth on cobalt is another notable advantage, resulting in the formation of high-quality epitaxial layers. The capability of Ag-coated Co to withstand brief exposures (duration of a few minutes) to ambient conditions while preserving the magnetic patterns is demonstrated in section S2 of the Supporting Information.
4.2. Irradiation at High $\mathrm{CO}$ Doses $\left(>10^{3} \mathrm{~L}\right)$. $\mathrm{CO}$ exposures of the order or in excess of $10^{3} \mathrm{~L}$ result in a much larger amount of carbon being deposited on the surface. As noted above and also confirmed by XPS, carbon initially forms carbides and then accumulates in a graphitic form. The diskshaped structure shown in Figure $4 \mathrm{a}$ was fabricated by irradiating a $5 \mathrm{AL}$ thick Co film with $60 \mathrm{eV}$ electrons at a $\mathrm{CO}$ partial pressure of $1 \times 10^{-6} \mathrm{mbar}$, delivering a total dose of $2.7 \times 10^{3} \mathrm{~L}$. After restoring UHV conditions, the specimen was heated at the rate of $\sim 1.35^{\circ} \mathrm{C} / \mathrm{s}$ up to a maximum temperature of about $380{ }^{\circ} \mathrm{C}$. During treatment, we monitored the film structure with the LEEM and observed a quick increase in the LEEM reflectivity at $8 \mathrm{eV}$ at temperatures above $320^{\circ} \mathrm{C}$. This behavior reflects not only an improved ordering within the $\mathrm{C}$ overlayer but also a chemical change, with carbon transforming from carbidic to graphitic type. The annealing treatment was stopped immediately after saturation of the LEEM intensity to avoid dewetting and minimize islanding in cobalt. The transformations occurring in the adlayer and film upon thermal treatment are summarized in Scheme 1.

The LEEM image in Figure 4a proves the sharp morphology of the pattern. Importantly, we do not observe the opening of "holes" inside the disk, or islanding outside the irradiated areas, indicating that the cobalt has not undergone a lateral rearrangement. This is confirmed by measurements of the low-energy electron reflectivity. As seen in Figure $4 b$, the nonirradiated areas exhibit nearly identical characteristics before and after the treatment. The observation of quantum 
Scheme 1. Diagram illustrating the transformation of the adlayer and Co film upon thermal treatment. (1) The nonirradiated, $\mathrm{CO}$-covered surface is annealed to or above $200{ }^{\circ} \mathrm{C}$ to desorb $\mathrm{CO}$ and recover the clean, as-grown surface. (2) The e-beam irradiated $\mathrm{C}$-covered surface is ramped to $380{ }^{\circ} \mathrm{C}$; carbidic $\mathrm{C}$ transforms into graphitic $\mathrm{C}$. Short thermal treatments were found to leave the morphology of the as-grown film unaffected, but prolonged annealing caused the formation of threedimensional Co islands in the nonirradiated surface regions. Islanding never occurs in the graphitized regions

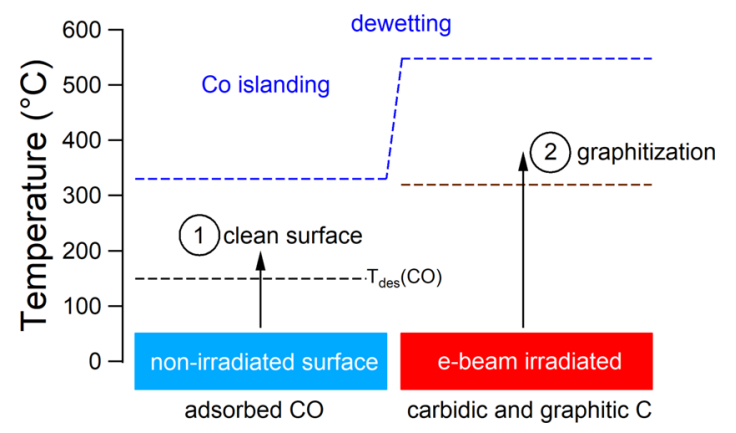

well resonances (see section S1 in the Supporting Information) indicates homogeneous characteristics across the surface and confirms that the initial thickness of $\sim 5 \mathrm{AL}$ is preserved. Prolonged annealing above $400{ }^{\circ} \mathrm{C}$ results instead in some dewetting and islanding outside of the irradiated areas, but not inside. Consistent with previous data on Co under graphene on $\operatorname{Ir}(111),{ }^{46}$ the C-covered regions always appear laterally homogeneous, which can be attributed to graphene effectively hindering Co diffusion and islanding. As we could verify by XPS, further annealing does not improve the quality of the graphitic layer.

XPEEM spectroscopy and imaging was used to locally assess the chemical state of a similarly fabricated microstructure. As can be seen from the spectra plotted in Figure 4c, the C 1s emission strongly differs inside and outside the disk. The inset within the graph represents an XPEEM map of the $C 1$ s emission and demonstrates well the accumulation of carbon inside the irradiated area. Within the disk, the $\mathrm{C} 1 \mathrm{~s}$ emission is peaked at $284.9 \mathrm{eV}$, confirming the graphitic nature of the adlayer. $^{39}$ In fact, the line shape and peak intensity (we estimate a coverage of $0.77 \mathrm{eML}_{\mathrm{gr}}$ ) compares well with what we recorded for CVD-grown ML graphene supported on ultrathin $\mathrm{Co} / \mathrm{Re}(0001)$ (also shown in the figure). Outside the disk, the $\mathrm{C} 1 \mathrm{~s}$ emission is considerably weaker, indicating that a much smaller amount of carbon is adsorbed on the surface, about $0.08 \mathrm{eML}_{\mathrm{gr}}$. The $\mathrm{C} 1 \mathrm{~s}$ peak is here centered at $283.5 \mathrm{eV}$, fingerprint of carbide formation. This signal likely originates from residual $\mathrm{CO}$ adsorption and subsequent dissociation upon soft X-ray irradiation. Remarkably, no emission from the $\mathrm{O}$ 1s core level was detected inside or outside the irradiated area (not shown in the figure), suggesting that any residual adsorbed oxygen reacts with carbon during thermal treatment. The graphitic nature of the adlayer is demonstrated by the $\mu$ ARPES data shown in Figure 4d and also confirmed by LEED. Both measurements indicate the formation of a rotationally incoherent graphene layer. Even if broadened, the $\pi$ band of carbon is clearly visible in the momentum distribution curves, permitting us to estimate a Dirac energy close to $2.6 \mathrm{eV}$, which is in good agreement with literature data for single-layer epitaxial graphene on $\mathrm{Co}(0001)^{47}$ and the closely related system of graphene on $\mathrm{Ni}(111){ }^{48}$

The magnetic state of the surface was investigated using XMCD-PEEM at the $\mathrm{Co}_{3}$ edge. Figure 5a,b shows XMCD-

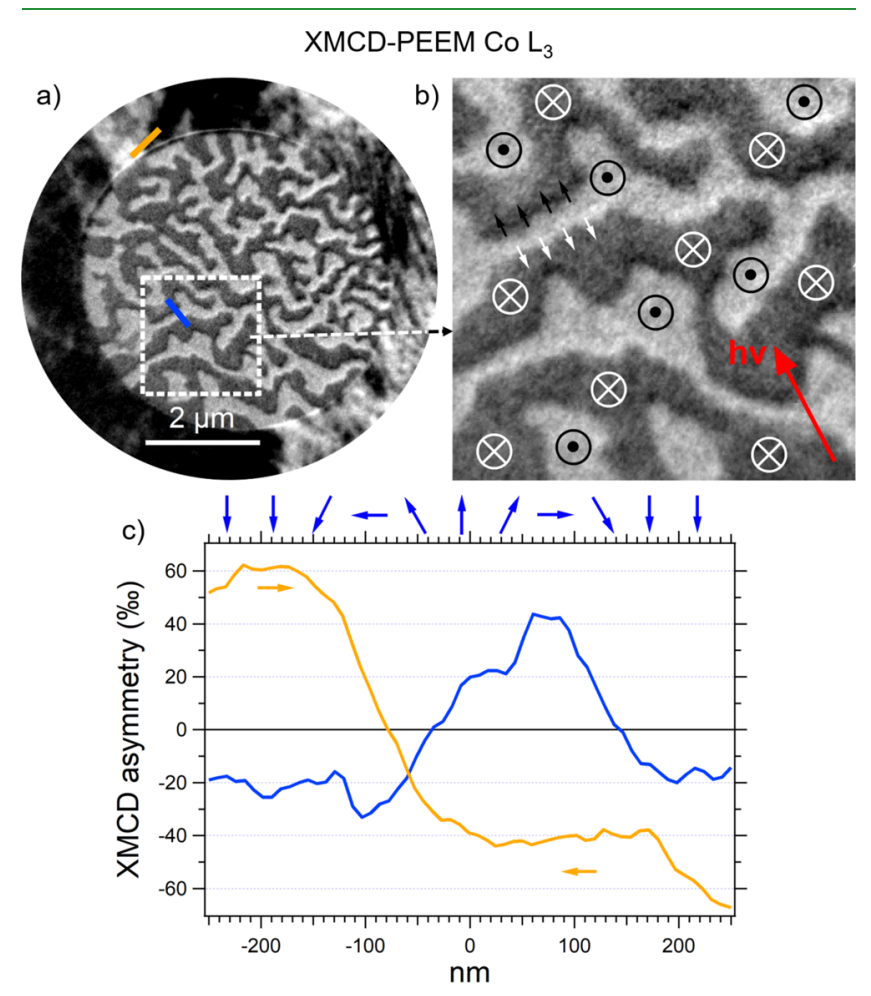

Figure 5. (a) $\mathrm{Co}_{3}$ edge XMCD-PEEM image of the same surface region shown in Figure 4a. The irradiated disk exhibits a stripe pattern, indicating out-of-plane MA. (b) Magnified image of the region highlighted by the white dashed square in (a). As can be seen, the image contrast increases at the walls between domains with opposite magnetization. The red arrow represents the direction of the photon beam; the other symbols indicate the orientation of the magnetization vector. (c) Cross-sectional intensity profiles taken along the blue and orange lines in (a). The blue curve illustrates the increase in dichroic contrast at the domain wall. The small black and white arrows mimic the orientation of the magnetization vector across the Neél-type domain wall. Chirality is right-handed. The orange contour exemplifies the larger image contrast that is observed in the regions exhibiting in-plane $\mathrm{MA}$.

PEEM images of the same structure shown in Figure 4a. Here, the contrast is purely magnetic and reveals a stripe pattern inside the irradiated area, confirming the enhancement of outof-plane MA already observed at low carbon coverage. The bright and dark gray regions correspond to domains with the magnetization pointing up and down from the film plane, respectively, as indicated by the symbols. The domain width has increased by a factor of about 3 compared to the case of low $\mathrm{CO}$ doses, suggesting that the magnetic state is slightly more distant from the SRT critical point. ${ }^{49}$ Note also that the out-of-plane domains are separated by narrow regions where the contrast is enhanced (pointed by arrows in the figure). This feature is well evidenced by the intensity profile shown in Figure 5c. Such increase in dichroic contrast occurs solely when the domain walls are oriented at $90^{\circ}$ from the X-ray beam. This means that the magnetization is aligned in-plane along the propagation direction of the photon beam, but perpendicular to the domain wall surface. As can be deduced 
from the arrows positioned in the top panel of Figure 5c, the walls are of Neél type. Also, we observe that the magnetic contrast in the domain wall is dictated by the orientation of the out-of-plane domains, that is, the domain walls are chiral. The observation of chirality reflects the occurrence of asymmetric DMI at the two interfaces of the film, a configuration that is known to occur in ternary superlattices ${ }^{50,51}$ and $\mathrm{MgO} / \mathrm{Co} / \mathrm{Pt}$ stacks. We note that the domain walls observed in graphene/ $\mathrm{Co} / \mathrm{Re}$ exhibit opposite chirality with respect to those seen in $\mathrm{MgO} / \mathrm{Co} / \mathrm{Pt},{ }^{52}$ which most likely points to the characteristics of the interaction with the different heavy metals. Nonetheless, graphene may have a crucial role in regulating the DMI, as has been recently proposed for graphene on $\mathrm{Co} / \mathrm{Ru} .^{18}$

To further investigate the magnetic properties of the graphene-coated Co, laterally resolved XMCD spectra were acquired for out-of-plane domains with opposite magnetization. The computation of the magnetic circular dichroism sum rules (see section S3 in the Supporting Information) permitted us to estimate the spin and orbital moments, $\mu_{\text {spin }}$ and $\mu_{\text {orb }}$, respectively, for which we found values of 1.7 and $0.16 \mu_{\mathrm{B}} /$ atom and the ratio $\mu_{\text {orb }} / \mu_{\text {spin }}=0.095$. Our results are in excellent agreement with literature data for bulk hcp cobalt, ${ }^{53}$ suggesting that the graphitic overlayer does not affect the atomic-scale magnetism of Co. Hence, we exclude the formation of a nonmagnetic Co topmost layer, consistent with previous reports for CO-covered Co films. ${ }^{23}$ In the present case, the PMA is most probably connected to electronic effects on the topmost Co layer after the chemisorption of atomic carbon. This interpretation is supported by previous work on the $\mathrm{CO} / \mathrm{Co} / \mathrm{Pd}(111)$ system, the occupation of specific adsorption sites being related to the occurrence of an inplane to out-of-plane SRT in Co. ${ }^{23,26}$ Graphene and varied carbon-based molecules can affect the MA of intercalated ferromagnetic layers in a similar manner. ${ }^{54,55}$ In particular, graphene is known to stabilize out-of-plane magnetization in Co films on $\operatorname{Ir}(111)$ up to a thickness of $25 \AA ̊$. Related to this, ab initio calculations demonstrate that this PMA enhancement is originated by the hybridization between Co $d_{z}{ }^{2}$ and $d_{y z}$ orbitals, which is in turn induced by carbonp states. ${ }^{1}$ Magneto-elastic effects are instead to be excluded, based on previous investigations on ultrathin Co films covered by adsorbates $^{23,26}$ or capped by noble metals. ${ }^{45}$ Clearly, our magnetic patterning method modifies the film magnetic state by forming chemical bonds at its vacuum interface. The film MA is thus controlled through interfacial effects, whereas the bulk remains structurally unaffected. This is a pronounced difference with ion irradiation patterning techniques, where the ions induce structural (e.g., disorder or a phase transition) or chemical transformations at much larger depths in the magnetic medium.

\section{CONCLUSIONS}

In conclusion, electron-stimulated dissociation of $\mathrm{CO}$ was employed to graft chemomagnetic micropatterns with sharp edges $(\sim 30 \mathrm{~nm})$ in hcp cobalt films with thickness in the range 4-5 AL. These patterns exhibit a C-rich surface and were found to induce PMA in the cobalt support, which has otherwise in-plane MA. The wavelength of out-of-plane striped magnetic domains was found to increase with the increasing amount of carbon deposited on the surface. Essentially, our method adds lateral control on the SRT process, enabling to write patterns of arbitrary shape. Thus, one can aim at spatially confining the magnetization direction by simply controlling the amount of carbon that is deposited on the surface. Related to this, our results suggest that the interpretation of the early ebeam patterning experiments should be reconsidered, taking into account the effects of beam-induced dissociation of adspecies containing carbon.

Most importantly, we demonstrated that prolonged e-beam irradiation in $\mathrm{CO}$ ambient results in the accumulation of the $\mathrm{ML}$ amount of carbon on the surface, which can be converted to graphene upon subsequent annealing at $380{ }^{\circ} \mathrm{C}$. We envisage several applications of our method in fundamental studies on magnetism. For instance, one can fabricate artificial heterostacks comprising different ferromagnetic layers, where the magnetic coupling is tuned by an interposed graphene spacer. ${ }^{17}$ Further work is clearly necessary to optimize conditions to grow atomically flat FM layers on graphene. Very promising results have already been produced in pulsed laser deposition experiments, which demonstrated close to perfect layer-by-layer growth for Co on graphene in the thickness range $3-5$ AL. ${ }^{56}$ As a first step in this direction, we focused here on graphene on ultrathin Co on Re, which we consider a prototypical building block for the fabrication of more complex stacks. We found that graphene enhances PMA in cobalt and, most interestingly, that the walls separating outof-plane domains are chiral of Neél type. This suggests that skyrmions can be nucleated and stabilized in the Co film under appropriate conditions. In this respect, the lithographic capabilities of our method are particularly appealing, as they permit to exploit confinement effects without destroying the lateral continuity of the Co film.

As a final point, we note that extreme ultraviolet radiation or soft X-rays can be used in place of electrons because CO molecular dissociation is also stimulated by photons. The use of masks or advanced e-beam lithography methods ${ }^{57}$ can further improve the lateral resolution as well as the printing speed, adding versatility and true scalability to the printing protocol.

\section{METHODS SECTION}

6.1. Spectroscopic Photoemission and LEEM. All experiments hereby described were carried out with the SPELEEM (ELMITEC $\mathrm{GmbH}$ ) at the "Nanospectroscopy" beamline of the Elettra storage ring in Trieste (Italy). In the LEEM operation of the microscope, the specimen is illuminated with a microfocused, collimated electron beam emitted by an $\mathrm{LaB}_{6}$ cathode. The beam diameter on sample is about $80 \mu \mathrm{m}$. The beam energy, varied in the range $0-750 \mathrm{eV}$, is regulated by applying a bias voltage (start voltage $V_{S}$ ) to the sample. Imaging is possible in either real or reciprocal space, with three illumination apertures permitting to restrict the e-beam footprint to a diameter of $5 \mu \mathrm{m}, 1 \mu \mathrm{m}$, or $500 \mathrm{~nm}$. When the beamline X-rays are used, the SPELEEM can implement laterally resolved versions of Xray absorption spectroscopy and related methods, namely, XMCDPEEM. Thanks to a bandpass energy filter, the SPELEEM also allows spectral imaging measurements (XPEEM), reaching lateral resolution below $30 \mathrm{~nm}$ and energy resolution of about $300 \mathrm{meV}$. Microprobe photoemission spectroscopy (e.g., $\mu$-XPS) operation is also possible, which enables fast acquisition and energy resolution as good as $\sim 110$ meV. ${ }^{19}$ These measurements are restricted to an area of about $2 \mu \mathrm{m}$ in diameter. Depending on the flux available, the BEs were referenced to the Fermi level or the Re $4 \mathrm{f}$ core-level emission from the bulk metal.

6.2. XMCD-PEEM. XMCD is based on the dependence of the Xray absorption intensity on the relative orientation of the magnetization and the X-ray polarization. In X-ray microscopy, this effect is used to image magnetic domains in thin ferromagnetic layers and nanostructures. ${ }^{21}$ The XMCD image intensity was calculated using 
the following algebra: $I_{\mathrm{XMCD}}=\frac{I_{-}-I_{+}}{I_{-}+I_{+}}$, where $I_{-}$and $I_{+}$represent the pixel intensities of the PEEM images acquired with negative and positive helicities of the photon beam. The image contrast is maximum when the photon helicity and the magnetization directions are parallel and anti-parallel. Because of the grazing incidence of the photon beam at an angle of $16^{\circ}$ with respect to the surface plane, the dichroic contrast recorded for in-plane magnetization is up to about 3.5 times larger than for the case of out-of-plane magnetization.

6.3. Sample Preparation. The $\operatorname{Re}(0001)$ single crystal (Surface Preparation Labs) was cleaned by $\mathrm{Ar}^{+}$sputtering at $2 \mathrm{kV}$ followed by repeated temperature cycling between 400 and $800{ }^{\circ} \mathrm{C}$ at an oxygen pressure of $2 \times 10^{-6}$ mbar. After the treatment, residual oxygen was desorbed with a flash to $1700{ }^{\circ} \mathrm{C}$ in UHV. Before experiments, no impurities could be detected in XPS. $\mu$-LEED on a single terrace showed a sharp $(1 \times 1)$ pattern with threefold symmetry. No evidence of oxygen-related half-order spots was seen, even diffuse ones. LEEM showed uniform contrast across terraces and no evidence of islanding, thus excluding the presence of contaminants.

6.4. Co Growth. All experiments were carried out at a base pressure of less than $1.5 \times 10^{-10}$ mbar. Co was dosed using a commercial e-beam evaporator (Omicron Focus EMF3), sublimated from high-purity rods (Goodfellow Ltd) at a maximum deposition rate of about $0.1 \mathrm{ML} / \mathrm{min}$. The specimen temperature was measured using a C-type thermocouple $\left(\mathrm{W}_{96} \mathrm{Re}_{4}-\mathrm{W}_{74} \mathrm{Re}_{26}\right)$, spot-welded on the Mo ring supporting the sample. The first Co layer was deposited at $350{ }^{\circ} \mathrm{C}$, which warrants step-flow growth conditions. In this manner, defect-free pseudomorphic Co MLs could be obtained. To achieve a uniform thickness of the Co film as well as prevent alloying with the Re substrate, the following layers were grown at room temperature, which results in the development of the $10 \times 10$ moire structure, indicating the relaxation of the lattice constant to the bulk value. ${ }^{58}$ Postgrowth annealing at $250{ }^{\circ} \mathrm{C}$ was carried out to reduce surface roughness. LEEM measurements in the dark-field mode demonstrated that, after thermal treatment, Co retains the hcp structure. ${ }^{59,60}$

\section{ASSOCIATED CONTENT}

\section{(S) Supporting Information}

The Supporting Information is available free of charge on the ACS Publications website at DOI: 10.1021/acsami.8b07485.

Methods for film thickness determination, capping layers for magnetic pattern preservation, and the XMCD magnetometry results for graphene/5ALCo/Re (PDF)

\section{AUTHOR INFORMATION}

\section{Corresponding Author}

*E-mail: andrea.locatelli@elettra.eu.

\section{ORCID}

Alessandro Sala: 0000-0002-5845-1301

Andrea Locatelli: 0000-0002-8072-7343

\section{Present Address}

${ }^{\perp}$ (A.S.) IOM-CNR Laboratorio TASC, S.S. $14 \mathrm{~km} 163.5$ in AREA Science Park, Basovizza, I-34149 Trieste, Italy.

\section{Notes}

The authors declare no competing financial interest.

\section{ACKNOWLEDGMENTS}

This work is part of the scientific activities of the CERIC-ERIC internal project MAG-ALCHEMI. We thank M. Kiskinova and E. Bauer for illuminating discussions and critical reading of this manuscript.

\section{REFERENCES}

(1) Sander, D.; et al. The 2017 Magnetism Roadmap. J. Phys. D: Appl. Phys. 2017, 50, 363001.
(2) Randolph, S. J.; Fowlkes, J. D.; Rack, P. D. Focused, Nanoscale Electron-beam-induced Deposition and Etching. Crit. Rev. Solid State Mater. Sci. 2006, 31, 55-89.

(3) Rupp, W.; Biedermann, A.; Kamenik, B.; Ritter, R.; Klein, C.; Platzgummer, E.; Schmid, M.; Varga, P. Ion-beam Induced fcc-bcc Transition in Ultrathin Fe Films for Ferromagnetic Patterning. Appl. Phys. Lett. 2008, 93, 063102.

(4) Zaman, S. S.; Dvořák, P.; Ritter, R.; Buchsbaum, A.; Stickler, D.; Oepen, H. P.; Schmid, M.; Varga, P. In-situ Magnetic Nanopatterning of Fe Films Grown on $\mathrm{Cu}(100)$. J. Appl. Phys. 2011, 110, 024309.

(5) Chappert, C.; Bernas, H.; Ferré, J.; Kottler, V.; Jamet, J.-P.; Chen, Y.; Cambril, E.; Devolder, T.; Rousseaux, F.; Mathet, V.; Launois, H. Planar Patterned Magnetic Media Obtained by Ion Irradiation. Science 1998, 280, 1919-1922.

(6) Bali, R.; Wintz, S.; Meutzner, F.; Hübner, R.; Boucher, R.; Ünal, A. A.; Valencia, S.; Neudert, A.; Potzger, K.; Bauch, J.; Kronast, F.; Facsko, S.; Lindner, J.; Fassbender, J. Printing Nearly-discrete Magnetic Patterns Using Chemical Disorder Induced Ferromagnetism. Nano Lett. 2014, 14, 435-441.

(7) Hlawacek, G.; Bali, R.; Röder, F.; Aleksandrov, Y.; Semisalova, A.; Wintz, S.; Wagner, K.; Schultheiss, H.; Facsko, S.; Fassbender, J.; et al. Tailoring Magnetic Nanostructures with Neon in the Ion Microscope. Microsc. Microanal. 2016, 22, 1716-1717.

(8) Kim, S.; Lee, S.; Ko, J.; Son, J.; Kim, M.; Kang, S.; Hong, J. Nanoscale Patterning of Complex Magnetic Nanostructures by Reduction with Low-energy Protons. Nat. Nanotechnol. 2012, 7, 567-571.

(9) Albisetti, E.; Petti, D.; Pancaldi, M.; Madami, M.; Tacchi, S.; Curtis, J.; King, W. P.; Papp, A.; Csaba, G.; Porod, W.; Vavassori, P.; Riedo, E.; Bertacco, R. Nanopatterning Reconfigurable Magnetic Landscapes via Thermally Assisted Scanning Probe Lithography. Nat. Nanotechnol. 2016, 11, 545-551.

(10) Allenspach, R.; Bischof, A.; Dürig, U.; Grütter, P. Local Modification of Magnetic Properties by an Electron Beam. Appl. Phys. Lett. 1998, 73, 3598-3600.

(11) Monchesky, T. L.; Unguris, J.; Celotta, R. J. Electron Beam Stimulated Spin Reorientation. J. Appl. Phys. 2003, 93, 8241-8243.

(12) Reeve, R. M.; Chin, S.-L.; Kopper, K.-P.; Ionescu, A.; Barnes, C. H. W. Experimental and theoretical study of electron-beam-induced spin-reorientation transition reversal in the $\mathrm{CO} / \mathrm{Co}\left(\begin{array}{lll}1 & 1 & 0\end{array}\right)$ system. J. Phys. D: Appl. Phys. 2012, 45, 275003.

(13) Reeve, R.; Chin, S.-L.; Kopper, K. P.; Ionescu, A.; Barnes, C. H. W. Electron Beam Tuning of the Magnetic Anisotropy in Co/ $\mathrm{Cu}(110)$ Films. IEEE Trans. Magn. 2011, 47, 1554-1558.

(14) Avouris, P.; Walkup, R. E. Fundamental Mechanisms of Desorption and Fragmentation Induced by Electronic Transitions at Surfaces. Annu. Rev. Phys. Chem. 1989, 40, 173-206.

(15) Ramsier, R. D., Jr.; Yates, J. T. Electron-stimulated desorption: Principles and applications. Surf. Sci. Rep. 1991, 12, 246-378.

(16) Yang, H.; Vu, A. D.; Hallal, A.; Rougemaille, N.; Coraux, J.; Chen, G.; Schmid, A. K.; Chshiev, M. Anatomy and Giant Enhancement of the Perpendicular Magnetic Anisotropy of CobaltGraphene Heterostructures. Nano Lett. 2016, 16, 145-151.

(17) Gargiani, P.; Cuadrado, R.; Vasili, H. B.; Pruneda, M.; Valvidares, M. Graphene-based Synthetic Antiferromagnets and Ferrimagnets. Nat. Commun. 2017, 8, 699.

(18) Yang, H.; Chen, G.; Cotta, A. A. C.; N’Diaye, A. T.; Nikolaev, S. A.; Soares, E. A.; Macedo, W. A. A.; Schmid, A. K.; Fert, A.; Chshiev, M. Significant Dzyaloshinskii-Moriya Interaction at Graphene-Ferromagnet Interfaces due to Rashba Effect. arXiv e-prints, 2017.

(19) Menteş, T. O.; Zamborlini, G.; Sala, A.; Locatelli, A. Cathode Lens Spectromicroscopy: Methodology and Applications. Beilstein J. Nanotechnol. 2014, 5, 1873-1886.

(20) Bauer, E. Surface Microscopy with Low Energy Electrons; Springer: New York, 2014; pp 409-464. 
(21) Cheng, X. M.; Keavney, D. J. Studies of Nanomagnetism Using Synchrotron-based X-ray Photoemission Electron Microscopy (XPEEM). Rep. Prog. Phys. 2012, 75, 026501.

(22) El Gabaly, F.; Gallego, S.; Muñoz, C.; Szunyogh, L.; Weinberger, P.; Klein, C.; Schmid, A. K.; McCarty, K. F.; de la Figuera, J. Imaging Spin-Reorientation Transitions in Consecutive Atomic Co Layers on $\mathrm{Ru}(0001)$. Phys. Rev. Lett. 2006, 96, 147202.

(23) Matsumura, D.; Yokoyama, T.; Amemiya, K.; Kitagawa, S.; Ohta, T. X-ray Magnetic Circular Dichroism Study of Spin Reorientation Transitions of Magnetic Thin Films Induced by Surface Chemisorption. Phys. Rev. B: Condens. Matter Mater. Phys. 2002, 66, 024402 .

(24) Yokoyama, T.; Matsumura, D.; Amemiya, K.; Kitagawa, S.; Suzuki, N.; Ohta, T. Spin Reorientation Transitions of Ultrathin Co/ $\operatorname{Pd}(111)$ Films Induced by Chemisorption: X-ray Magnetic Circular Dichroism Study. J. Phys.: Condens. Matter 2003, 15, S537-S546.

(25) Matsumura, D.; Amemiya, K.; Kitagawa, S.; Shimada, T.; Abe, H.; Ohta, T.; Watanabe, H.; Yokoyama, T. Surface Structure of CO on $\operatorname{CoPd}(111)$ Magnetic Thin Films and its Effect on the Spin Reorientation Transition of the Film. Phys. Rev. B: Condens. Matter Mater. Phys. 2006, 73, 174423.

(26) Matsumura, D.; Nakagawa, T.; Watanabe, H.; Abe, H.; Amemiya, K.; Ohta, T.; Yokoyama, T. Magnetization process of $\mathrm{Co} / \mathrm{Pd}(111)$ thin films: Chemisorption-induced spin-reorientation transition. Surf. Sci. 2008, 602, 1999-2003.

(27) Dzyaloshinsky, I. Thermodynamic Theory of Weak Ferromagnetism of Antiferromagnetics. J. Phys. Chem. Solids 1958, 4, 241-255.

(28) Moriya, T. Anisotropic Superexchange Interaction and Weak Ferromagnetism. Phys. Rev. 1960, 120, 91-98.

(29) Duden, T.; Bauer, E. Magnetization Wrinkle in Thin Ferromagnetic Films. Phys. Rev. Lett. 1996, 77, 2308-2311.

(30) Itikawa, Y. Cross Sections for Electron Collisions with Carbon Monoxide. J. Phys. Chem. Ref. Data 2015, 44, 013105.

(31) Ramaker, D. E. Comparison of photon stimulated dissociation of gas phase and chemisorbed CO. J. Chem. Phys. 1983, 78, 29983013.

(32) Lambert, R. M.; Comrie, C. M. The Role of Primary and Secondary Electrons in Electron Induced Desorption and Dissociation: CO on Pt(111). Surf. Sci. 1973, 38, 197-209.

(33) Lahtinen, J.; Vaari, J.; Kauraala, K. Adsorption and Structure Dependent Desorption of CO on Co(0001). Surf. Sci. 1998, 418, 502-510.

(34) Gong, X.-Q.; Raval, R.; Hu, P. CO Dissociation and O Removal on Co(0001): a Density Functional Theory Study. Surf. Sci. 2004, $562,247-256$.

(35) Allenspach, R.; Bischof, A. Magnetization Direction Switching in $\mathrm{Fe} / \mathrm{Cu}(100)$ Epitaxial Films: Temperature and Thickness Dependence. Phys. Rev. Lett. 1992, 69, 3385-3388.

(36) Kashuba, A.; Pokrovsky, V. L. Stripe Domain Structures in a Thin Ferromagnetic Film. Phys. Rev. Lett. 1993, 70, 3155-3158.

(37) As can be seen in Figure 2f, the dichroic contrast recorded outside the irradiated disk is up to a factor $\sim 3.5$ times larger than inside. This value matches the expected contrast difference for inplane and out-of-plane magnetization for an incidence angle of the photon beam on the sample of $16^{\circ}$, as occurs in our set-up. Note also that the XMCD contrast in the stripe pattern is independent from the stripe orientation, which further confirms that the magnetization is oriented out-of-plane in the irradiated areas.

(38) Wang, H.; Wong, S. P.; Cheung, W. Y.; Ke, N.; Wen, G. H.; Zhang, X. X.; Kwok, R. W. M. Magnetic Properties and Structure Evolution of Amorphous Co-C Nanocomposite Films Prepared by Pulsed Filtered Vacuum Arc Deposition. J. Appl. Phys. 2000, 88, 4919-4921.

(39) Preobrajenski, A. B.; Ng, M. L.; Vinogradov, A. S.; Mårtensson, N. Controlling Graphene Corrugation on Lattice-Mismatched Substrates. Phys. Rev. B: Condens. Matter Mater. Phys. 2008, 78, 073401.
(40) Günther, S.; Kolmakov, A.; Kovac, J.; Kiskinova, M. Artefact Formation in Scanning Photoelectron Emission Microscopy. Ultramicroscopy 1998, 75, 35-51.

(41) Denley, D.; Perfetti, P.; Williams, R. S.; Shirley, D. A.; Stöhr, J. CarbonK-edge fine structure in graphite foils and in thin-film contaminants on metal surfaces. Phys. Rev. B: Condens. Matter Mater. Phys. 1980, 21, 2267-2273.

(42) Chauvet, C.; Polack, F.; Silly, M. G.; Lagarde, B.; Thomasset, M.; Kubsky, S.; Duval, J. P.; Risterucci, P.; Pilette, B.; Yao, I.; Bergeard, N.; Sirotti, F. Carbon contamination of soft X-ray beamlines: dramatic anti-reflection coating effects observed in the 1 $\mathrm{keV}$ photon energy region. J. Synchrotron Radiat. 2011, 18, 761-764.

(43) Lahtinen, J.; Vaari, J.; Taio, A.; Vehanen, A.; Hautojärvi, P. Adsorption and Desorption Measurements of $\mathrm{CO}$ and $\mathrm{O} 2$ on Cobalt. Vacuum 1990, 41, 112-114.

(44) Nowitzki, T.; Carlsson, A. F.; Martyanov, O.; Naschitzki, M.; Zielasek, V.; Risse, T.; Schmal, M.; Freund, H.-J.; Bäumer, M. Oxidation of Alumina-Supported Co and Co-Pd Model Catalysts for the Fischer-Tropsch Reaction. J. Phys. Chem. C 2007, 111, 85668572.

(45) El Gabaly, F.; McCarty, K. F.; Schmid, A. K.; de la Figuera, J.; Muñoz, M. C.; Szunyogh, L.; Weinberger, P.; Gallego, S. Noble Metal Capping Effects on the Spin-reorientation Transitions of Co/ $\mathrm{Ru}(0001)$. New J. Phys. 2008, 10, 073024.

(46) Rougemaille, N.; N’Diaye, A. T.; Coraux, J.; Vo-Van, C.; Fruchart, O.; Schmid, A. K. Perpendicular magnetic anisotropy of cobalt films intercalated under graphene. Appl. Phys. Lett. 2012, 101, 142403.

(47) Usachov, D.; Fedorov, A.; Otrokov, M. M.; Chikina, A.; Vilkov, O.; Petukhov, A.; Rybkin, A. G.; Koroteev, Y. M.; Chulkov, E. V.; Adamchuk, V. K.; Grüneis, A.; Laubschat, C.; Vyalikh, D. V. Observation of Single-Spin Dirac Fermions at the Graphene/ Ferromagnet Interface. Nano Lett. 2015, 15, 2396-2401.

(48) Varykhalov, A.; Marchenko, D.; Sánchez-Barriga, J.; Scholz, M. R.; Verberck, B.; Trauzettel, B.; Wehling, T. O.; Carbone, C.; Rader, O. Intact Dirac Cones at Broken Sublattice Symmetry: Photoemission Study of Graphene on Ni and Co. Phys. Rev. X 2012, 2, 041017.

(49) Kashuba, A. B.; Pokrovsky, V. L. Stripe domain structures in a thin ferromagnetic film. Phys. Rev. B: Condens. Matter Mater. Phys. 1993, 48, 10335-10344.

(50) Chen, G.; Schmid, A. K. Imaging and Tailoring the Chirality of Domain Walls in Magnetic Films. Adv. Mater. 2015, 27, 5738-5743.

(51) Chen, G.; Mascaraque, A.; N’Diaye, A. T.; Schmid, A. K. Room Temperature Skyrmion Ground State Stabilized through Interlayer Exchange Coupling. Appl. Phys. Lett. 2015, 106, 242404.

(52) Boulle, O.; et al. Room-temperature Chiral Magnetic Skyrmions in Ultrathin Magnetic Nanostructures. Nat. Nanotechnol. 2016, 11, 449-454.

(53) Chen, C. T.; Idzerda, Y. U.; Lin, H.-J.; Smith, N. V.; Meigs, G.; Chaban, E.; Ho, G. H.; Pellegrin, E.; Sette, F. Experimental Confirmation of the X-Ray Magnetic Circular Dichroism Sum Rules for Iron and Cobalt. Phys. Rev. Lett. 1995, 75, 152-155.

(54) Coraux, J.; N'Diaye, A. T.; Rougemaille, N.; Vo-Van, C.; Kimouche, A.; Yang, H.-X.; Chshiev, M.; Bendiab, N.; Fruchart, O.; Schmid, A. K. Air-Protected Epitaxial Graphene/Ferromagnet Hybrids Prepared by Chemical Vapor Deposition and Intercalation. J. Phys. Chem. Lett. 2012, 3, 2059-2063.

(55) Bairagi, K.; Bellec, A.; Repain, V.; Chacon, C.; Girard, Y.; Garreau, Y.; Lagoute, J.; Rousset, S.; Breitwieser, R.; Hu, Y.-C.; Chao, Y. C.; Pai, W. W.; Li, D.; Smogunov, A.; Barreteau, C. Tuning the Magnetic Anisotropy at a Molecule-Metal Interface. Phys. Rev. Lett. 2015, 114, 247203

(56) Vo-Van, C.; Kassir-Bodon, Z.; Yang, H.; Coraux, J.; Vogel, J.; Pizzini, S.; Bayle-Guillemaud, P.; Chshiev, M.; Ranno, L.; Guisset, V.; David, P.; Salvador, V.; Fruchart, O. Ultrathin Epitaxial Cobalt Films on Graphene for Spintronic Investigations and Applications. New J. Phys. 2010, 12, 103040.

(57) Manfrinato, V. R.; Stein, A.; Zhang, L.; Nam, C.-Y.; Yager, K. G.; Stach, E. A.; Black, C. T. Aberration-corrected Electron Beam 
Lithography at the One Nanometer Length Scale. Nano Lett. 2017, 17, 4562-4567.

(58) Parschau, M.; Christmann, K. Cobalt on rhenium(0001) - an example of thermally activated layer intermixing and surface alloying. Surf. Sci. 1999, 423, 303-323.

(59) de la Figuera, J.; Puerta, J. M.; Cerda, J. I.; El Gabaly, F.; McCarty, K. F. Determining the structure of $\mathrm{Ru}(0001)$ from lowenergy electron diffraction of a single terrace. Surf. Sci. 2006, 600, L105-L109.

(60) El Gabaly, F.; Puerta, J. M.; Klein, C.; Saa, A.; Schmid, A. K.; McCarty, K. F.; Cerda, J. I.; de la Figuera, J. Structure and morphology of ultrathinCo/Ru(0001) films. New J. Phys. 2007, 9, 80. 